10 minutes after extubation. Attenuation of airway response and Extubation quality was rated using extubation quality scale. Adverse effects, Extubation and Emergence time were recorded. Results: All data measured and analyzed using standard statistical tests (student's t-test, Chi-square, Fisher's Exact Probability tests, Man- Whitney, Wilcoxon rank test). A ' $P$ ' value of $\leq 0.05$ was taken as significant. The systolic, diastolic blood pressure and heart rate were higher in group $\mathrm{C}$ than in group $\mathrm{D}$. The need for rescue medications (nitroglycerine for hypertension, esmolol for tachycardia) was higher in group C. Extubation quality scores were superior in group D than group C. Extubation time and Emergence time were comparable in both the groups. Conclusion: Dexmedetomidine in a single dose of $0.25 \mathrm{mcg} / \mathrm{kg}$ is safe and efficacious in attenuation of hemodynamic responses and airway reflexes during extubation following spine surgery without prolonging emergence and extubation times.

\section{Effect of tranexamic acid on blood loss and quality of surgical field in trans-sphenoidal surgery}

\section{Amlan Swain, Jyotsna Wig, Nidhi Bidyut Panda, Ritesh Lamsal, K. K. Mukherjee ${ }^{1}$ Department of Anesthesiology and ${ }^{1}$ Neurosurgery PGIMER,
Chandigarh, India}

Background: The aim of this study is to assess the effect of preoperative bolus followed by intraoperative infusion of tranexamic acid on intraoperative blood loss and the quality of surgical field in trans-sphenoidal surgery. Materials and Methods: A prospective randomized double blind study was carried out in 50 ASA grade I-II patients presenting for elective trans-sphenoidal pituitary surgery for pituitary tumors. One group received bolus intravenous dose of tranexamic acid $(25 \mathrm{mg} / \mathrm{kg})$ followed by infusion $(1 / \mathrm{mg} / \mathrm{kg} / \mathrm{hr})$ of the same till the end of surgery while the placebo group received normal saline in the same amount. All patients were analyzed for preoperative color perception, intraoperative hemodynamic monitoring, hematocrit, arterial blood gas values, quality of surgical field (Boezaart scale), amount of blood loss and anaesthetic requirements. Results: The demographic, hemodynamic and anesthetic parameters, duration of surgery, amount of intraoperative fluid and urine output were comparable in both the groups. The tranexamic group had significantly better quality of surgical field (Boezaart score $2.64 \pm 0.638$ vs. $3.08 \pm 0.759$, $P=0.031)$ and lesser blood loss (334 \pm 101 vs. $495 \pm 226 \mathrm{ml}$, $P=0.02)$ than the placebo group. Conclusions: Despite widespread use in other surgical specialities, tranexamic acid is rarely used in neurosurgical operations. In standard doses, it is found to be effective in traumatic brain injury and spinal surgery. Therefore, unless contraindications exist, tranexamic acid can be tried in neurosurgery. Conclusion: Intraoperative use of tranexamic acid improves the quality of surgical field as well as reduces bleeding in patients undergoing trans-sphenoidal resection of pituitary tumors.

\section{Effect of dexmedetomidine as anaesthetic adjuvant in patients undergoing trans-sphenoidal pituitary surgery}

\author{
Renu Bala, Arvind Chaturvedi, Mihir P. Pandia, \\ Parmod K. Bithal \\ Department of Neuroanaesthesiology, AIIMS, New Delhi, \\ India
}

Background: We conducted a prospective, randomized, double-blind, placebo-control study to evaluate use of dexmedetomidine as anaesthetic adjuvant in patients undergoing trans-sphenoidal pituitary surgery. We tried to find its effect in attenuating hemodynamic response to nasal speculum insertion and on early postoperative recovery profile and intra operative consumption of anaesthetic agents. Materials and Methods: After obtaining approval from ethics committee, we enrolled 60 adult patients of either sex belonging to ASA-status I and II planned to undergo elective trans-sphenoidal pituitary surgery. Routine investigations and preoperative assessment was done as a standard protocol and injection glycopyrrolate $0.2 \mathrm{mg}$ intramuscularly was administered $30 \mathrm{~min}$ prior to surgery. In group D $(n=30)$ dexmedetomidine $1 \mathrm{mcg} / \mathrm{kg}$ over 10 minutes followed by $0.5 \mathrm{mcg} / \mathrm{kg} / \mathrm{hr}$ was given in infusion while in group C $(n=30)$ normal saline ran in the same rate. Induction of anesthesia was achieved with sleep dose of propofol, fentanyl $2 \mathrm{mcg} / \mathrm{kg}$ and rocuronium $1 \mathrm{mg} / \mathrm{kg}$. Monitoring for heart rate, ECG, non-invasive and invasive blood pressure, oxygen saturation, end-tidal carbon di-oxide, BIS, along with neuromuscular monitoring was carried out. Peri-operative hemodynamic parameters were recorded and any fluctuation (increase or decrease) were treated as per protocol. The total dose of anesthetics, analgesics, muscle relaxant, and other drugs (anti-hypertensive's) used were noted. The duration of anesthesia, surgery, nasal speculum duration and intraoperative fluids, blood loss and urine output were also recorded. Any adverse event or complication if occurred was noted. After conclusion of surgery, neuromuscular blockade was reversed and recovery profile (emergence time, extubation, time for visual examination, time for first analgesic, modified aldrete score, VAS score, Ramsay sedation score, postoperative nausea and vomiting and shivering) were recorded. The data was compiled and analyzed using SPSS for Windows version 20. Results: Our study demonstrated that patient in dexmedetomidine group had significant reduction in hemodynamic response to nasal 
speculum insertion and had perioperative hemodynamic stability. There was reduction in anaesthetic, analgesic, muscle relaxant consumption in group D. Emergence time and extubation time was less, and there were fewer episodes of postoperative nausea and vomiting and shivering in group D. VAS was less and time for first analgesic was prolonged in patients of dexmedetomidine group. There were few incidences of side-effects like bradycardia and hypotension. Conclusion: We conclude that dexmedetomidine as anesthetic adjuvant is efficacious in attenuating nasal speculum response, provides intraoperative hemodynamic stability, decreases intraoperative anaesthetic requirement and hastens early recovery without any serious side-effects.

\section{Miracle of a syringe attached to the pilot balloon of endotracheal tube during neurosurgical procedures}

\section{Karthik Raj, M. V. Niranjan Kumar, A. Rout \\ Department of Neuroanesthesia, Sri Ramachandra Medical College and Hospital, Porur, Chennai, Tamil Nadu, India}

Background: The use of cuff inflating syringe in situ results in preventing airway related complications during neurosurgical procedures in various positions. Materials and Methods: It is a prospective study including all neurological surgeries done under GA from 2000. The parameters noted were duration of anesthesia and a standard $10 \mathrm{ml}$ of air is used to inflate the cuff to prevent air leak, the remaining amount of air in syringe is noted and the amount of air left in situ is noted at the end of procedure. Results: In $20 \%$ of patients there was an increase in air volume of which 5-10\% increase was noted in $2 \mathrm{hrs}, 20-25 \%$ in $2-4 \mathrm{hrs}$ and up to $50 \%$ for surgeries lasting more than 5-6 hrs. We had 5 patients of which 3 patients who had air leak during posterior cervical surgeries and 2 patients had complete tube blockade at bevel end of the ET tube. Conclusion: Securing and maintaining the ET tube in long duration procedures and positions other than supine can be difficult as the patency of tube can be compromised also the added risk of using nitrous oxide which can diffuse into the cuff increasing its volume and pressure. Complications like tube blockade at bevel end, hoarseness, laryngeal edema, vocal cord edema, accidental extubation and ventilator malfunction can be prevented. The simple technique of leaving the cuff inflating syringe can help to avoid airway catastrophe as the airway is inaccessible during the procedure.

\section{Anesthesia for carotid stenting: Our experience}

\section{Deepa Chaturvedi}

Department of Anaesthesia, Neuroanesthesia unit, Max Super specialty hospital, Saket, New Delhi, India

Background: Patients with carotid artery disease frequently have other co-morbidities especially cardiac disease. A new option of treatment, carotid artery stenting (CAS) shows lower risk of stroke and myocardial infraction but high incidence of hemodynamic changes during procedure. We reviewed the anesthetic management and complications during and after treatment of CAS. Materials and Methods: One hundred and fourteen patients who had CAS over a period of four years from 2009-2013 were reviewed. For conventional common femoral artery approach procedure was done under conscious sedation with midazolam and fentanyl.Oxygen was supplied with nasal prongs. The groin site was anesthetized with local infiltration of lidocaine. Monitoring include ECG, invasive BP, SpO2. After femoral sheath insertion temporary pacemaker lead was placed and attached to the pacemaker. Prophylactic vagolytic agent atropine $0.6 \mathrm{mg}$ IV was used prior to balloon inflation to block the baroreceptor response. Heparin was given before placement of carotid stent. Result: Most of the patients were ASA Grade 3. Common pre-procedure morbidities were Coronary Artery Disease, HT, DM and peripheral vascular disease along with neurological deficits. 12 patients had TIA, 10 with stroke and 1 patient had aphasia. One patient had femoral artery tear at the puncture site, which was secured by Perclose-intra arterial suture. Conclusion: Anesthesia for carotid intervention requires protection of the brain from ischemic insult. Attention to physiological factors influencing cerebral blood flow is mandatory. The anesthesiologist plays a crucial role in maintaining hemodynamic stability, adjusting anticoagulation and monitoring neurological status.

\section{Comparison of hemodynamic responses to intubation: Flexible fibreoptic bronchoscope versus McCoy laryngoscope in presence of rigid cervical collar simulating cervical immobilization for traumatic cervical spine}

Nitesh Gill, Shobha Purohit

Department of Anaesthesia, S M S Medical College, Jaipur, Rajasthan, India

Background: Intubation is known to cause exaggerated hemodynamic response in form of tachycardia, hypertension and dysrrhythmias. In cervical spine immobility or instability, intubation has to be performed using cervical immobilization to prevent 